\title{
Session 6: Discussion on Propeller Systems
}

\author{
Domitilla de Martino \\ INAF - Astronomical Observatory of Capodimonte, Via Moiariello 16, \\ I-80131, Naples, Italy
}

\begin{abstract}
I summarize some aspects which have been raised for discussion during Session 6, devoted to magnetic propeller systems.
\end{abstract}

\section{Introduction}

The session on propeller systems was devoted to the unique magnetic CV, AE Aqr, which contains the fastest rotating $\left(\mathrm{P}_{\text {rot }}=33 \mathrm{~s}\right)$ white dwarf among Intermediate Polars in a wide orbit $\left(\mathrm{P}_{\mathrm{orb}}=9.88 \mathrm{~h}\right)$. The rapidly spinning and moderately strong magnetic field is believed to prevent the formation of an accretion disc and material is expelled from the system.

Since the first dedicated session during the past Cape Workshop on MCVs, many have been the theoretical and observational efforts to understand the strong flaring activity and the link between accretion and outflow processes in this peculiar CV.

Presentations on new evolutionary paths, on theory of flares and observations on a wide energy range, from radio to X-rays, have brought further insights into the current understanding of this complex system.

\section{Discussion}

The magnetic propeller model, proposed by Wynn, King \& Horne in 1997, is able to account for the observed spin-down of the white dwarf, energetics of flares and to broadly reproduce the optical Doppler tomograms. However a concern has been raised during the discussion by Coel Hellier, who points out on the existence of a large number of tomograms, further confirmed by Gaghik Tovmassian, which are not all conclusive for the propeller model. In contrast to this, Martin Still replies that the majority of them are broadly in line with that model. It has to be recognized, however, that quantitative predictions of the model are still missing and, hence, a detailed comparison of this model with the observations has still to be performed.

Further improvements have been done in the context of modeling flares at optical and radio wavelengths, as presented by Kevin Pearson and Luis Venter respectively. The latter explains how about twenty expanding synchroton emitting blobs can account for the observed integrated radio flux during outbursts. Related to this aspect, Steve Howell asks about the distance from the source reached by the blobs. Martin Still replies that the VLBI image is extended and 
Meil Abada-Simon further confirms this, reporting that the radio source is resolved up to a radius of about four times the binary separation. Another point is raised by Frederic Hessmann, on the fact that if blobs cannot be resolved, the flux and density should be low. Pieter Meintjes replies that densities are $\sim 10^{14}-10^{15} \mathrm{~cm}^{-3}$.

The ejected material has been also searched via a complex and innovative analysis of HST images which has been presented by Martin Still. Whether the total mass of the circumstellar material has been measured, has been questioned by Klaus Beuermann. Martin Still replies that it has not been done yet. Related to this, Klaus Schenker points out that the mass loss rate can be measured from the nebula.

From this session, it appears that a step forward has been done gathering new observational constraints for further detailed modeling in the near future. For instance, the new XMM-Newton observations, presented by Julian Osborne, show X-ray velocities not easy to reconcile with the propeller model. Hence, the complex interplay of accretion and ejection in such a magnetized rapidly rotating white dwarf still deserves further study to be fully understood. 